\title{
Konkurrenzschutzvereinbarungen im Krankenhaus Wie sicher sind sie?
}

Verstößt ein Krankenhaus gegen eine vertraglich vereinbarte Konkurrenzschutzklausel, kann der Betroffene ggf. Unterlassung verlangen (so Urteil des OLG München vom 10.11.2010, Az.: 20 U 2514/10).

\section{Der Fall}

Der Kläger, ein Facharzt für Urologie, hatte mit der Klinik unter anderem einen Nutzungsvertrag mit fester Laufzeit bis zum 31.12.2017 vereinbart. Zwischen den Parteien wurden über die Jahre mehrere Verträge abgeschlossen. Es handelte sich zunächst um einen Belegarztvertrag, später um einen Kooperationsvertrag und schließlich um besagten Nutzungsvertrag. Die Präambel des Nutzungsvertrages enthielt eine Absichtserklärung, wonach die Zusammenarbeit intensiviert und die urologische Praxis in das Klinikum integriert werden sollte. Darüber hinaus nahm die Präambel sowohl auf die belegärztliche Tätigkeit des Klägers als auch auf den Kooperationsvertrag Bezug. Der Nutzungsvertrag sah eine sogenannte Konkurrenzschutzklausel vor, in der vereinbart war, dass für die Laufzeit dieses Vertrages die Klinik Verträge mit anderen Fachärzten für Urologie nur nach vorheriger Zustimmung durch den Kläger abschließen würde. Während der Laufzeit des Vertrages wurde der Kläger von der Klinik darüber informiert, dass sie beabsichtige, mit einem anderen Arzt einen Beleg- und/oder Konsiliararztvertrag abzuschließen. Dieser Vertrag mit dem Dritten kam auch dann in der Folgezeit zustande. Der Kläger forderte die Klinik auf, den Vertrag mit dem weiteren Urologen nicht fortzusetzen, was von der Klinik jedoch abgelehnt wurde.

Der Kläger verwies auf die Konkurrenzschutzklausel und vertrat die Auffassung, die Klinik dürfe Beleg- oder Konsiliarärzte der Fachrichtung Urologie nur mit seiner Zustimmung in ihrem Klinikum beschäftigen. Er beantragte daher, der Beklagten bei Androhung von Ordnungsgeld zu verbieten, Beleg- oder Konsiliarärzte der Fachrichtung Urologie ohne seine vorherige Zustimmung in ihrem Klinikum zu beschäftigen. Die Beklagte beantragte Klageabweisung mit dem Argument, dass der Konkurrenzschutz sich lediglich auf den Abschluss von Nutzungsverträgen mit anderen Ärzten der Fachrichtung Urologie beziehe und nicht als umfassender Konkurrenzschutz verstanden werden könne.
Das Landgericht Landshut gab dem Antrag des Klägers zunächst in vollem Umfang statt. Die Konkurrenzschutzklausel des Nutzungsvertrages gewähre dem Kläger den geltend gemachten Unterlassungsanspruch, da die Parteien hiermit einen umfassenden Konkurrenzschutz geregelt hätten. Dem Konsiliararztvertrag der Klinik mit dem weiteren Arzt habe der Kläger nicht zugestimmt. Die geäußerte Weigerung der Klinik, die Zusammenarbeit mit diesem Arzt zu beenden, begründet die berechtigte Annahme der Gefahr einer anderen Verletzung der Konkurrenzschutzklausel auch durch den Abschluss weiterer Verträge mit anderen Urologen.

Gegen dieses Urteil ging die Klinik in Berufung und begehrte weiterhin Klageabweisung. Sie argumentiert damit, dass ein umfassender Konkurrenzschutz kartellrechtlich unzulässig und entsprechend unwirksam wäre. Das Oberlandesgericht München bestätigte in zweiter Instanz im Wesentlichen das Urteil des LG Landshut. Es stellte allerdings fest, dass der Kläger zwar einen Anspruch auf die von ihm begehrte Unterlassung habe, jedoch nicht auf unbestimmte Zeit, sondern nur bis zur Beendigung des Nutzungsvertrages.

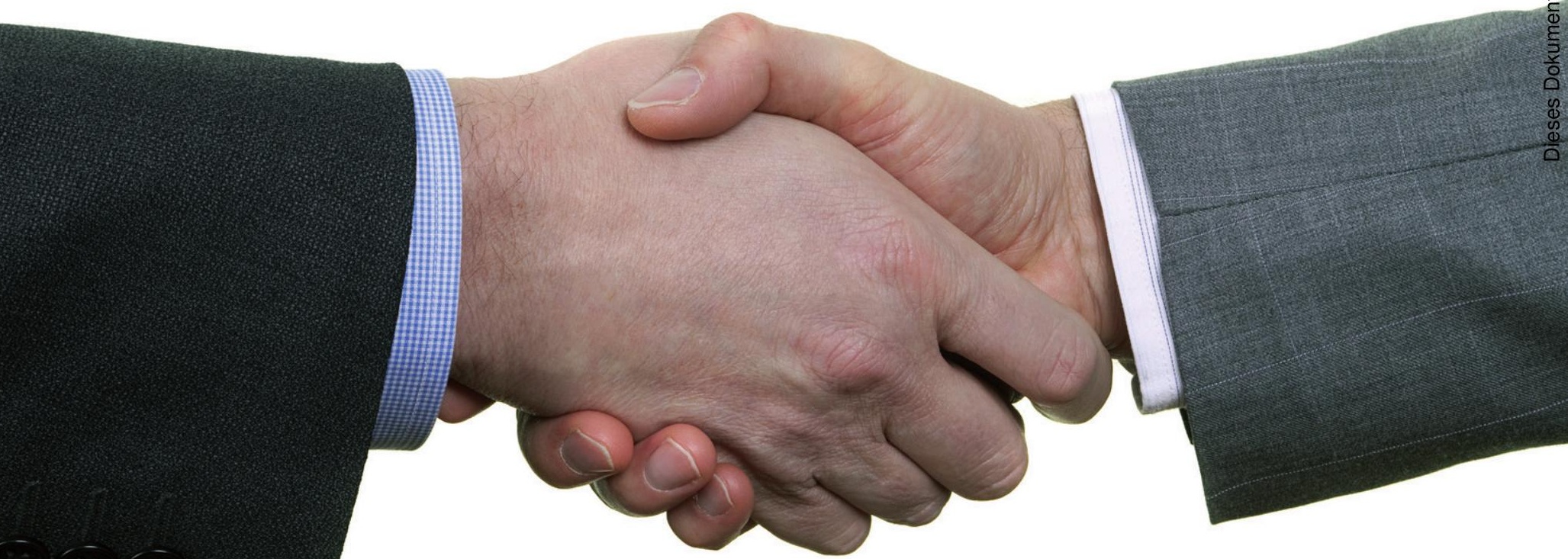




\section{Gesamtkontext der Vertragslage} entscheidend

Aufgrund der Bezugnahme auf die vorherigen Verträge in der Präambel des Nutzungsvertrages vertrat das OLG die Auffassung, dass die Konkurrenzschutzklausel des Nutzungsvertrages im Gesamtkontext der zwischen den Parteien abgeschlossenen Verträge zu betrachten sei. Im Rahmen der Auslegung sei entscheidend, was bei Abschluss des Nutzungsvertrages gewollt gewesen sei. Auf dieser Grundlage sei der Senat der Meinung, dass die Klinik gemäß der Konkurrenzschutzklausel des Nutzungsvertrages nicht nur Nutzungs-, sondern auch sämtliche andere Verträge mit Fachärzten für Urologie nur nach vorheriger Zustimmung des Klägers abschließen darf. Unter Berücksichtigung der im gesamten Vertragswerk aufgebauten herausragenden und leitenden Funktion des Klägers in der urologischen Abteilung könne nur ein umfassendes Zustimmungserfordernis gemeint gewesen sein, mit welchem der Kläger Einfluss auf die Zusammensetzung des unter seiner fachlichen Leitung stehenden Ärzteteams nehmen konnte.

\section{Einzelfallbetrachtung erforderlich}

Nach Auffassung der Richter verstößt die Konkurrenzschutzregelung auch nicht gegen das Kartellrecht. Das Vertragsverhältnis zwischen den Parteien bestand bei der Vereinbarung des Konkurrenzschutzes im Wesentlichen in einer Kooperationsvereinbarung zur Führung der urologischen Abteilung und in einem Nutzungsvertrag über Praxisräume. Es handele sich also um ein Austauschvertragsverhältnis mit einer inhaltlich auf weitere Fachärzte der Urologie begrenzten Konkurrenzschutzklausel. Eine Wettbewerbsbeschränkung in diesem Sinne sei zulässig, wenn für die vereinbarte Beschränkung bei wertender Betrachtungsweise im Hinblick auf die Freiheit des Wettbewerbs ein anzuerkennendes Interesse bestehe. Nach der Rechtsprechung sei anerkannt, dass Wettbewerbsverbote in gewerblichen Mietverträgen regelmäßig als selbstständige Nebenpflicht im Sinne von Treu und Glauben geboten seien. Entscheidend für die Annahme vertragsimmanenter Wettbewerbsverbote sei dabei die Verpflichtung von Vermietern gewerblich genutzter Räume - auch ohne Bestehen einer ausdrücklichen vertraglichen Regelung -, den jeweiligen Mieter gegen Konkurrenz im selben Haus zu schützen. Diese Rechtsprechung beruhe auf der Erwägung, dass es bei der Vermietung von Räumen zum Betrieb eines bestimmten Geschäftes zur Gewährung des vertragsgemäßen Gebrauchs gehöre, in anderen Räumen des Hauses oder auch unmittelbar angrenzenden Grundstücken des Vermieters kein Konkurrenzunternehmen zuzulassen. Unumstritten wie dieser Grundsatz selbst sei seine Eingrenzung dahingehend, dass der Vermieter nicht gehalten sei, dem Mieter jeden fühlbaren oder unliebsamen Wettbewerb fernzuhalten, vielmehr sei nach den Umständen des einzelnen Falles abzuwägen, inwieweit nach Treu und Glauben unter Berücksichtigung der Belange der Parteien die Fernhaltung von Konkurrenz geboten sei. Die Beschränkung dürfe nicht über die anerkennenswerten Interessen der Beteiligten hinausgehen. Ob ein solches anerkennenswertes Interesse vorläge, hänge davon ab, ob die Wettbewerbsbeschränkung (hier für weitere Fachärzte der Urologie am Klinikum der Beklagten) zur Erzielung eines gerechten Interessenausgleichs im Rahmen des jeweiligen Vertrages gerechtfertigt sei. Dies sei durch eine Gesamtwürdigung des Vertrages und seinen tatsächlichen Hintergrund zu beurteilen.

\section{Arzt hat berechtigtes Interesse an Konkurrenzschutz}

Unter Berücksichtigung aller Umstände sah der Senat ein solches berechtigtes Interesse im vorliegenden Fall als gegeben an. Die Beklagte hatte zum Zeitpunkt des Abschlusses des Nutzungsvertrages ein starkes Interesse daran, den bereits seit Jahren bei ihr als Belegarzt tätigen Kläger zur Optimierung der Patientenversorgung im Bereich der Urologie auch räumlich und darüber hinaus auch kooperativ in ihr Klinikum zu integrieren. Dies sei sowohl der Präambel des Kooperationsvertrages wie auch der Präambel des Nutzungsvertrages zu entnehmen. $\mathrm{Zu}$ diesem Zweck sollte der Kläger u.a. seine außerhalb des Klinikums befindliche Praxis in die Räume des Klinikums verlegen, als leitender Arzt der urologischen Belegabteilung des Klinikums fungieren und auf ärztlicher Seite die Verantwortung für die urologische Station und die Einhaltung der vereinbarten Kooperation übernehmen. Vor diesem Hintergrund gewähre die Konkurrenzschutzklausel dem Kläger nach dem Verständnis des Senats das Recht, dass Verträge mit anderen Fach- ärzten für Urologie nur nach seiner vorherigen Zustimmung geschlossen würden. Dies sei ein gerechter Interessenausgleich zur Aufgabe der Praxis außerhalb des Klinikums und der übernommenen Verpflichtung, die urologische Station auf ärztlicher Seite leistungsfähig zu erhalten. Die Konkurrenzschutzklausel erweise sich deshalb als funktionsnotwendig und zur Wahrung der beiderseitigen wirtschaftlichen Interessen als sachlich geboten.

Lediglich in zeitlicher Hinsicht sei die Unterlassungsverpflichtung gegenüber dem angefochtenen Urteil zeitlich $\mathrm{zu}$ beschränken. Die Konkurrenzschutzklausel des Nutzungsvertrages gewähre Konkurrenzschutz ausdrücklich nur für die Laufzeit des Vertrages.

\section{Fazit}

Das Urteil macht deutlich, dass besondere Sorgfalt bei der Erstellung der Verträge walten muss. Sollte der Konkurrenzschutz nicht umfassend gewollt sein, empfiehlt es sich, dies ausdrücklich vertraglich festzuhalten, um später nicht im Rahmen von Auslegungsschwierigkeiten die Frage streitig vor Gericht klären zu müssen. Die Problematik der Konkurrenzklauseln stellt sich häufig sowohl bei Beleg-, Konsiliar- als auch Nutzungsverträgen.

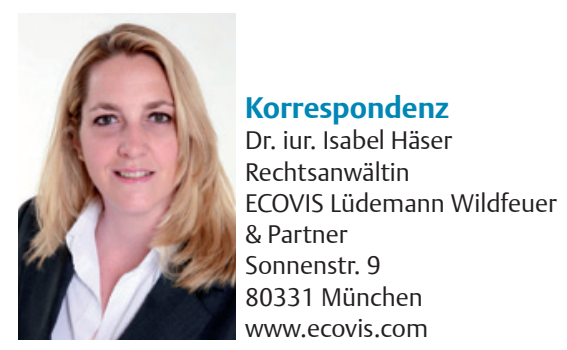

